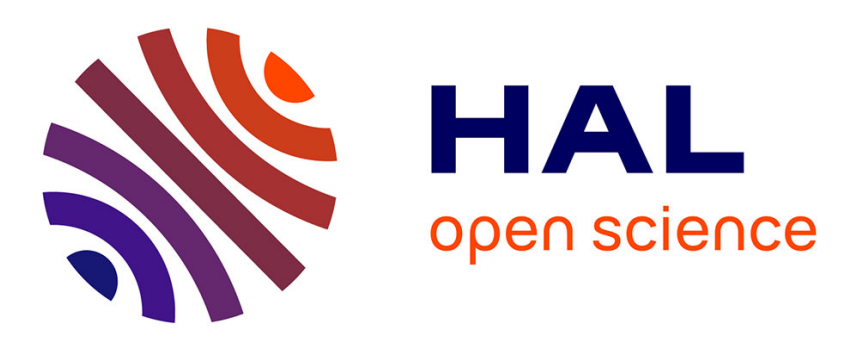

\title{
A Design Framework for Instrumenting Analytic Provenance for Problem-Solving Tasks
}

Lingxue Yang, Pierre Morizet-Mahoudeaux, Anne Guenand, Assia Mouloudi

\section{To cite this version:}

Lingxue Yang, Pierre Morizet-Mahoudeaux, Anne Guenand, Assia Mouloudi. A Design Framework for Instrumenting Analytic Provenance for Problem-Solving Tasks. 7th International Conference on Kansei Engineering and Emotion Research 2018 (KEER 2018), Mar 2018, Kuching, Sarawak, Malaysia. pp.633-643, 10.1007/978-981-10-8612-0_66 . hal-01790540

\section{HAL Id: hal-01790540 \\ https://hal.science/hal-01790540}

Submitted on 21 Jun 2021

HAL is a multi-disciplinary open access archive for the deposit and dissemination of scientific research documents, whether they are published or not. The documents may come from teaching and research institutions in France or abroad, or from public or private research centers.
L'archive ouverte pluridisciplinaire HAL, est destinée au dépôt et à la diffusion de documents scientifiques de niveau recherche, publiés ou non, émanant des établissements d'enseignement et de recherche français ou étrangers, des laboratoires publics ou privés. 


\title{
A design framework for instrumenting analytic provenance for problem-solving tasks
}

\author{
Lingxue Yang ${ }^{1,3}$, Pierre Morizet-Mahoudeaux ${ }^{1}$, Anne Guénand ${ }^{2}$, Assia Mouloudi $^{3}$ \\ ${ }^{1}$ Sorbonne-Universités, Université de Technologie de Compiègne \\ UMR CNRS 7253 HEUDIASYC \\ CS 60319, 60203 Compiègne-Cedex, France \\ lingxue.yang@hds.utc.fr; pierre.morizet@utc.fr \\ ${ }^{2}$ Sorbonne-Universités, Université de Technologie de Compiègne \\ Laboratoire COSTECH \\ CS 60319, 60203 Compiègne-Cedex, France \\ anne.guenand@utc.fr \\ ${ }^{3}$ SAP, 35, rue d'Alsace, 75098 Paris, France \\ lingxue.yang@sap.com; assia.mouloudi@sap.com
}

\begin{abstract}
In the context of analytics applications, the recall of interaction history often happens when users are identifying the root causes of a given problem based on a visual analytics task, which can be interrupted or suspended. The research of analytic provenance focuses on retrieving users' interaction history, reinstating their reasoning process so that they can quickly resume an interrupted or suspended task. Although many visualization analytic tools are available, they lack extended capabilities for giving access to users' interaction history in a natural coupling with their actions. We propose a design framework for instrumenting analytic provenance in a mode allowing users to "re-commit" to their tasks. We realize a first experiment to see how one's history activities has an impact on the way he/she resolves the task. We investigate the interaction possibilities of two design approaches: the user interface (UI) design in which the history path is considered as "put down" in the environment; the user experience (UX) design considers it as a coupling device between the user and the world, being "in hand" mode. The first part of our analysis shows that users use the history path for supporting their reasoning process. However, the indirect coupling between users' actions and provenance function keeps them outside of the history path so that they cannot easily link it to their current problem. We hypothesize that the "in hand" mode of interaction history will allow a natural coupling between a user's action and the provenance function, which may lead to a positive user experience. We then propose the lines for designing dynamic history path interaction tools.
\end{abstract}

Keywords: analytic provenance, UX design, design of a coupling device, perception, history path 


\section{Introduction}

In visual analytics tasks, users adopt root cause analysis strategy to identify the causal factors to a question. They get used to keeping track of what they did for testing hypothesis or generating results. In addition, when a project requires multiple analysis sessions, which last long periods of time, the task can be interrupted or suspended. Users try to find from "how did I get here?" to "where have I been" to reinstate their reasoning process. As our working memory is limited in its capacity and decay (Cowan, 2009), remembering and tracking the history becomes a barrier for exploration and commitment. Therefore, it is beneficial to design tools that help people recall their interaction history, and to get a positive user experience.

There has been a lot of research focusing on the history of analytic process for understanding the reasoning process through visualization interactions, which is called analytic provenance (North et al., 2011). Many visualization tools have been realized to help people recall their memory through analytic provenance (Bavoil et al., 2005; Dunne, Henry Riche, Lee, Metoyer, \& Robertson, 2012; Heer, Mackinlay, Stolte, \& Agrawala, 2008; Kadivar et al., 2009; Nguyen et al., 2016). Despite these advances, there is a scarcity on how to represent users' interaction history in a natural coupling with the current user action.

One opportunity to address the problem from a new perspective is to consider Charles Lenay's sensory-motor coupling, which addresses the two types of interaction between human and world (Lenay et al., 2007). Lenay et al. proposed two modes of existence of the perceptual interfaces: the "put down" mode, where the tool is perceived in the environment, and the "in hand" mode where the tool is considered as an organ, which gives extension to the possibilities of action. Even there are many visualization tools, they are typically in the "put down" mode, where to see one's own interaction history requires the user to navigate on a history panel or page which is statically represented. These proposals are not well adapted to commit users to memory recall. Thus, our aim is to propose a design framework for instrumenting analytic provenance in the "in hand" mode for motivating users to re-commit in the tasks that have been interrupted or suspended and to improve the task performance consequently.

To build our approach, we set up a first experiment - an experimental Tangram system - which records the user's interaction history. Observing from this experiment, we found that users had different and personal strategies of using the history path considered in a "put down" mode. This gave us an insight to rethink about the design of the "in hand" history path so as to allow users to go back to the interaction history to explore the dynamic of their previous attempts of resolution. We hypothesize that the "in hand" mode of interaction history, which will allow a natural coupling between a user's action and the provenance function, may lead to a positive user experience.

\section{Related Work}

The research of analytic provenance often focuses on the history capturing, representing and its supported operations, which are appropriate for users to recall discontinued 
tasks. We are interested in the visual aspects and interaction techniques to support analytic provenance, which evoke users' awareness in problem-solving tasks. In this part, we introduce related work in this area, focusing on how we will shape our contribution.

\subsection{Analytic provenance}

Shneiderman (1996) noted that tools enabling the display of users' interaction history supporting iterative analysis allow users to review, retrieve and revisit visualization states in the information visualization process. Since then, there has been more research on interaction history in analytics, HCI and other fields. Heer, Mackinlay, Stolte, and Agrawala (2008) summarized a range of design decisions in designing interaction history system. They include the organization of history analysis using states and actions, the visualization and the operations it supports. From the first workshop in 2011, the analytic provenance has been introduced to discuss its utilization in assisting users in solving real world problems (North et al., 2011).

Current provenance tools typically use the textual or graphic representation organized in linear sequence, branch, network layout, etc., which can be called out in a separate interface. They are supported by a set of operations on the history such as navigation, editing, sharing, etc. However, the coupling of user action and provenance function is indirect in terms of the time and space consistency. The provenance function can provide the information of "what have I done?" ("put down" mode), but doesn't make them aware of "what hasn't been done?" and "what should I do next?" using our perceptual system ("in hand" mode). In the next section, we present the difference between these two modes of analytic provenance, and highlight the benefits of using analytic provenance as a tool in the "in hand" mode.

\subsection{Lenay et al.'s sensory-motor coupling}

Our approach is based on the sensory-motor coupling proposed by Lenay et al. (2007). This theory illustrates the interaction between human and world, based on perception (Gibson, 1966) and phenomenology theory (Merleau-Ponty, 1945). The object can be alternatively perceived in the environment and as a coupling device between a subject and the world, thus modifying one's perception of the world. These two kinds of perception give rise to the modes of existence of an object: the "put down" and "in hand" modes, which help us understand the modes of analytic provenance in the interaction. An object in the "in hand" mode is used and grasped by a person to extend his or her capabilities to act and feel. For example, we wear our glasses, and we see the world through these glasses. However, we cannot perceive our glasses at this moment, they disappear from our consciousness. In this mode, the object is a tool that we seize to perceive the world. Whereas an object in the "put down" mode does not transform our perception, it can be modified, exchanged, etc. In the previous example, when we put down the glasses to clean and repair them, we can perceive them in the environment. These two modes alternate with each other in the utilization cycles of the object.

During a problem-solving task, existing analytic provenance displayed in the interface only indicates the history of the past actions, which can be perceived and acted on as in 
the "put down" mode. However, the analytic provenance corresponding to users' actions, which reinstates their previous reasoning process and makes them aware of unexplored areas, can be designed as a coupling device. It extends their capability of perceiving the problem-solving as a supplementation of their perceptual systems. In order to give users new possibilities of actions through new possibilities of perception of their analytic provenance, we propose to set up an experiment where the analytic provenance is designed as a coupling device. We first set up a minimalist experiment based on Tangram game.

\section{Tangram Experimentation}

Inspired by the efficiency of images for cueing autobiographical memory or memory about one's past (Lamming \& Newman, 1992), we integrated users' interaction history as a series of videos in the problem-solving context, which we called the history path. This experiment is aimed at evoking a discussion based on user behavior and investigating the design possibility.

\subsection{Procedure and tasks}

The experiment was carried out on a set of 18 participants ( 9 females, 9 males), most of them being company employees. We developed a web-based prototype based on Tangram game. The rule of Tangram is to build a given shape (in our case a rectangular isosceles triangle) using all the given pieces without overlapping each other. Participants used the mouse to play the game. The test consisted of a pre-test and a post-test. The pre-test was for collecting the interaction history that was used in the post-test. A Tobii eye tracker X2-30 was installed on the laptop to detect the participants' eye movements between the history path window and the tangram game window. The two sessions recorded the eye movements, screen, video and audio. Note that in this experiment, we were not interested whether the participants succeeded in the task, but how they possibly adapted their strategies during the problem-solving process.

Pre-test: Participants were introduced the test objective and confidential issues. Before the formal test, they were given 1 minute to be familiar with the prototype, and then we calibrated the eye tracker. After all setup, participants were given 10 minutes to try to resolve the problem (See Fig.1: pre-test). They were also asked to fill a questionnaire for their profile. Finally, we asked the participants not to search for the solution in anyway until the next test.

Post-test: 7 or 10 days later, we invited the same participants to do tangram test again. A history path of the videos in chronological order which captured their past actions in the pre-test (See Fig.1: post-test) was available. Each video was chosen based on their attempts to compose a kind of basic shape (which we name "a composition"). The cover of each video is the last frame of the video. 

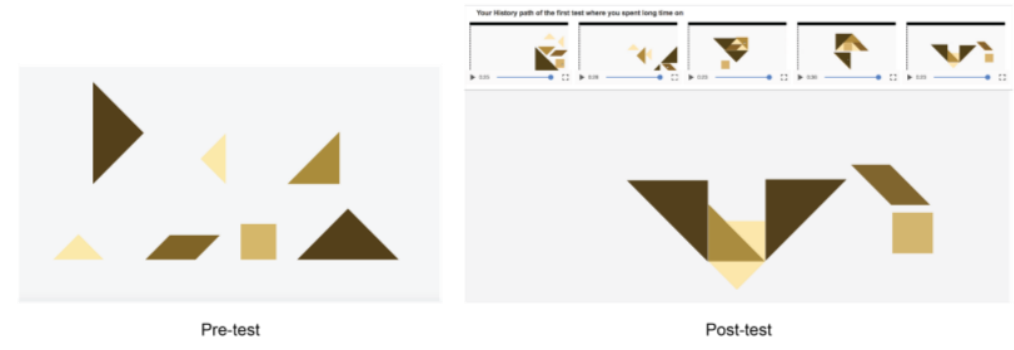

Fig. 1. Tangram games in pre-test, and post-test with history path

\subsection{Result}

We first analyzed the eye gaze video in the post test, normalized the way they interacted with the history path (HP) in three categories: simply scan the HP as a static image, replay the HP and NA (no action to the HP). We did the same with what they did after the interaction with the HP in two categories: they repeated the composition in the HP or they avoided the composition in the HP). The X-axis in Fig. 2 represents the number of participants; the blue, the orange, the gray and the yellow bars represent respectively the number of participants who avoided to rebuild the same compositions as in the pretest, those who repeated the same compositions, those who first repeated then avoid doing the same compositions and those who first avoided after looking at HP then repeated without seeing HP.

Among the 18 participants, 4 did not look at the HP (NA) while doing the task in the Post test (NA). Among the remaining 14 participants, 4 just scanned the HP and 10 participants replayed the HP. Within the 4 participants who scanned the HP, 3 did avoid what they did before. For the 10 who replayed, 5 did avoid, 3 first repeated then avoided and 2 first avoided then repeated.

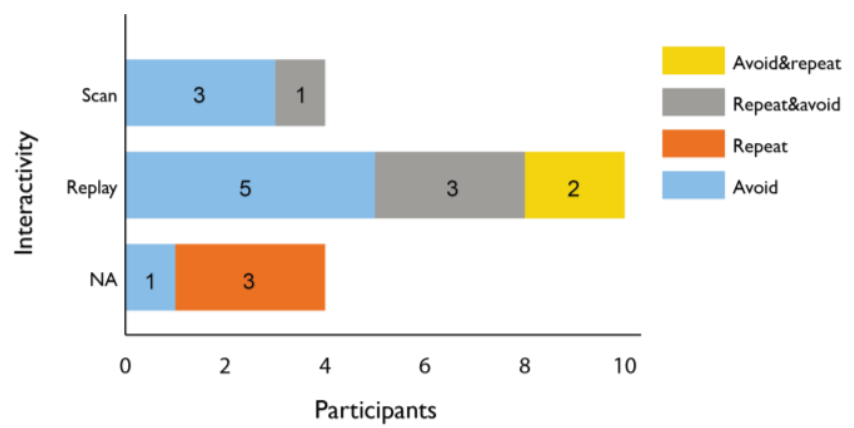

Fig. 2. Number of participants' reaction to their interactivity to the HP during the Post test

The gaze plot detected by the eye tracker (See Fig. 3) shows that the participants who replayed the HP spent more time looking at the content in the video (See Fig. 3: A) than the participant who just scanned it (See Fig. 3: B). This is indicated by their 
number of fixations and the duration of fixations. This gave us an insight of the correspondence between the eyes movements and an aid for measuring the commitment in the interaction.

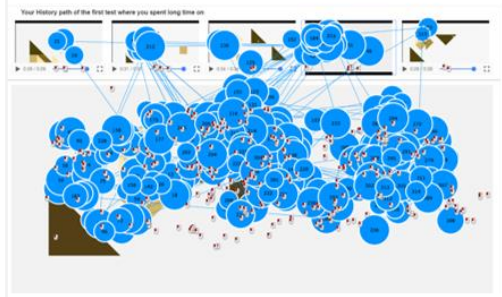

A

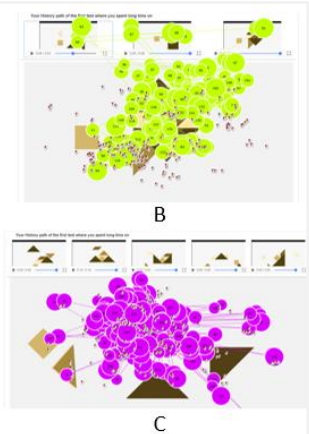

Fig. 3. Example of the gaze plot: A. replayed HP; B. scanned HP; C. No action on HP (NA)

The data we used are their number of fixations (fixation count) and the duration of fixations (fixation duration). As we can see in Table 1, the average of the fixation count in the history path in replay $(28,20 \mathrm{~s})$ is twice more than the one in scan $(12,75 \mathrm{~s})$. And the average of total fixation duration in the history path in replay $(42,26 \mathrm{~s})$ is four times longer than the one in scan (10,94 s). This gave us an insight of the correspondence between the eyes movements and an aid for measuring the commitment in the interaction.

Table 1. Gaze data for participants who played (top) and who scanned the HP (bottom)

\begin{tabular}{llll}
\hline $\begin{array}{l}\text { Participants } \\
\text { (replay) }\end{array}$ & $\begin{array}{l}\text { Fixation } \\
\text { Count }\end{array}$ & $\begin{array}{l}\text { Fixation Duration } \\
\text { Mean (seconds) }\end{array}$ & $\begin{array}{l}\text { Fixation } \\
\text { Duration } \\
\text { (seconds) }\end{array}$ \\
\hline P20 & 15 & 3,71 & 55,72 \\
P33 & 10 & 2,72 & 27,2 \\
P34 & 33 & 0,88 & 29,17 \\
P36 & 24 & 2,01 & 48,33 \\
P42 & 54 & 0,88 & 47,59 \\
P46 & 47 & 1,52 & 71,28 \\
P49 & 40 & 1,54 & 61,42 \\
P51 & 16 & 1,75 & 28,02 \\
P52 & 23 & 0,96 & 22,17 \\
P54 & 20 & 1,58 & 31,68 \\
Mean & $\mathbf{2 8 , 2 0}$ & 1,76 & $\mathbf{4 2 , 2 6}$ \\
\hline
\end{tabular}

\begin{tabular}{llll}
\hline $\begin{array}{l}\text { Participants } \\
\text { (scan) }\end{array}$ & $\begin{array}{l}\text { Fixation } \\
\text { Count }\end{array}$ & $\begin{array}{l}\text { Fixation Duration } \\
\text { Mean (seconds) }\end{array}$ & $\begin{array}{l}\text { Fixation } \\
\text { Duration } \\
\text { (seconds) }\end{array}$ \\
\hline P28 & 29 & 3,71 & 28,51 \\
P31 & 1 & 2,72 & 1,21 \\
P37 & 10 & 0,88 & 9,28 \\
P45 & 11 & 0,43 & 4,76 \\
Mean & $\mathbf{1 2 , 7 5}$ & $\mathbf{1 , 9 4}$ & $\mathbf{1 0 , 9 4}$ \\
\hline
\end{tabular}




\subsection{Discussion}

We can observe that displaying the HP constitutes a sign that involves the participants. Effectively, as 10 out of 18 replayed, 4 scanned and only 4 did not look at the HP. However, despite 14 out of 18 looked at the HP, the HP considered as a coupling device is not involving enough to allow participants to grasp their previous strategy and to build a new one based on these considerations. In addition, although we are mainly interested in their strategies to solve the problem rather than the success of the task, from the post questions, the success or failure did influence their impression on the HP.

The participants who avoided repeating what they did in the pre-test stated that the HP was useless because they failed. Therefore, the HP served as a reference both to avoid the same compositions and to verify if a new composition was already done in the pre-test. There are two reasons for the repetition: some found certain compositions matching their current idea, then they tried it; some repeated unconsciously when they did not look at the HP. Many participants expressed that seeing the HP was a waste of time and could distract them from the current task, given that they had only 10 minutes. However, if they felt close to the answer, they would have looked at the HP.

Above all, the participants tried to avoid consciously or repeat unconsciously the same attempts they had done in the pre-test. The avoidance behavior indicates that the HP serves as a support for their reasoning process. In addition, when the participants played the video, to some extent, they were engaged in the action-feedback loop. As shown in the eye movement of Fig. 3 and Table 1, people focused longer in the video when they played than when they just scanned the static image. We can infer that direct coupling between action and function is realized by this kind of demonstration. However, it is not a direct coupling between the user's current task and the HP. Besides, the HP is static, the participants had to work a lot to understand what they had done before and compare it with what they were doing now. In this case, the HP is still in the "put down" mode, which did not help enough the user to get a perception of the world they built and to get a positive user experience.

We hypothesize that the "in hand" mode of the HP will allow a natural coupling with user action and that the use of provenance function may lead to a positive user experience. In the next step, we will focus on the design of the history path in the "in hand" mode. It is aimed at making users understand in perceiving their successive attempts and exploring other possibilities, thus improving the task performance consequently.

\section{Second Experiment and New Design Proposal}

In order to design a dynamic HP in the "in hand" mode, we consider its visual representation as well as the accompanied interaction. In this section, we propose a design direction that we are going to implement in the next experiment to verify the hypothesis.

\subsection{Procedure and tasks of the History path design process}

From the first Tangram experiment, we observed that each participant had his or her own strategy for solving the problem (See Fig. 4). For example, some started from the 
big triangle, some from the square, some started from visioning the top of a triangle, etc. However, the experiment struggled to reinstate their previous reasoning process just by presenting the history path chronologically. The resulting questions was linked to how we define the history in Lenay et al.'s model of interaction.

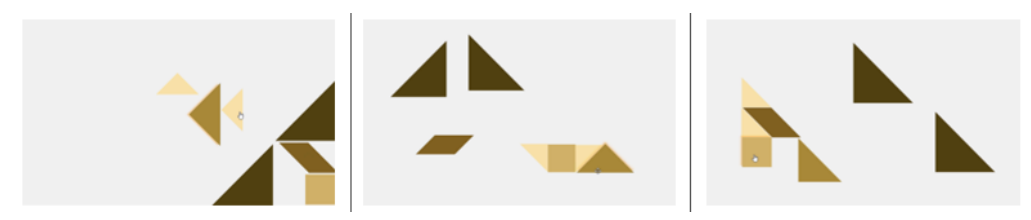

Fig. 4. Example of strategies of three participants

In the second Tangram experiment, we propose to design the HP as more involving, through its continuous enrichment along the task. We consider encoding users' strategy by computing their interaction history using certain data. We aim at identifying the patterns that we can display in order to assist users in realizing their deadlock and conducting another exploration. We record users' attempts of composition, and display similar attempts dynamically based on the user's current action. In terms of representation, we choose a tree-like diagram, where each branch starts from a composition of at least three shapes. For example, two triangles plus a square is a composition. When the user adds another triangle, we will display three triangles plus a square as a part of the branch. However, when the user removes a square and replaces it with a diamond, then another composition is created, enriching the history path as common core and branches (See Fig. 5: right).

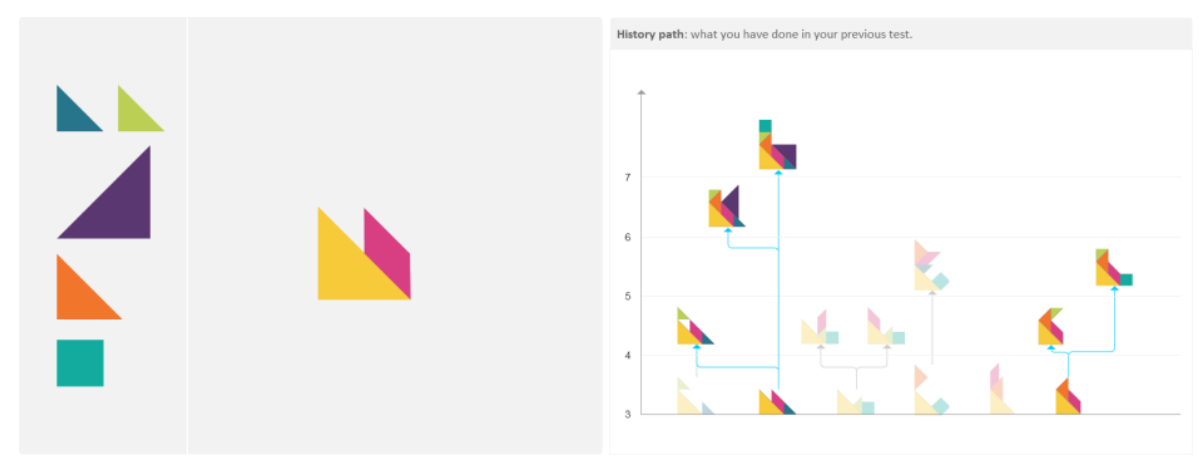

Fig. 5. Design proposal: history path (right) dynamically displayed based on user's action (left).

As shown in Fig. 5, each branch of the HP starts from a composition the position of which is based on the distance between the composition similarities. The branch which corresponds to the user's current action will be fully displayed, otherwise, the branch will be semi-transparent. The branch which consists of the exact user's current action will be displayed in blue. In this way, users can experience each exploration reflected from the HP's branch. The resulting HP coupling device allows to build new actions 
based on the perception of the results of their exploration and the proximity of previous exploration.

\subsection{Results}

As we are conducting this experiment we are gathering the results in order to analyze the user commitment in this second experiment, and compare it to the previous one.

\subsection{Discussion}

The goal of the study is to determine the effect of the natural coupling between the user's action and interaction history in a problem-solving context. In this respect, we conduct a controlled two-step experiment comparing a static HP where the history is chronologically displayed as in the first experiment, and a dynamic HP where an evolving tree graphics is graspable, as in the second experiment.

In the controlled experiment, each participant has been assigned to either a static path or a dynamic path, randomly distributed. In addition to recording eyes movements, screens, videos and audios, we compute the position of the components of a composition to have a dynamic trace of how the components are linked (by edges, vertices, ...) and how some were added or removed. Eye tracking is an unobtrusive method to collect users' eye movement data. We also use a retrospective think-aloud method, where the users are asked to elaborate on what they were doing and thinking during the test while looking at their gaze video (Pernice \& Nielsen, 2009).

Regarding to the evaluation method, we use both subjective and objective measures. Subjective measures are based on the semantic analysis such as developed by Osgood, Suci, \& Tannenbaum (1957) to evaluate the subjective impression on the HP. The participants are asked about their perception about the interaction quality based a set of adjective descriptors that we obtained from a previous study (Yang, Morizetmahoudeaux, Guénand, \& Mouloudi, 2016). For objective measures, we include the eye tracking metrics, the time spent on the task, the numbers of success. A comparison analysis between the static and dynamic history is carried out to test the hypothesis. As we are conducting the experiment, we are gathering the results in order to analyze user commitment in this second experiment, and compare to the previous experiment.

\section{Conclusion}

In this paper, we present the necessity to rethink the design approach for re-committing users in accessing their interaction history when the task has been interrupted or suspended. We propose to design the history path as a coupling device based on the current analytic provenance research and Lenay et al.'s theory. We set up a first Tangram experiment which allows to distinguish both design approaches: UI as static design of objects in the environment and the UX design of the history path as a coupling device. It gave us insight for a second experiment which analysis is under process, aiming at 
evaluating the effectiveness and positive user experience of a history path designed as coupling device compared to static object.

\section{References}

1. Bavoil, L., Callahan, S. P., Crossno, P. J., Freire, J., Scheidegger, C. E., Silva, C. T., \& Vo, H. T. (2005). VisTrails: Enabling interactive multiple-view visualizations. Proceedings of the IEEE Visualization Conference, (May 2014), 18. http://doi.org/10.1109/VIS.2005.113

2. Cowan, N. (2009). What are the differences between long-term, short-term, and working memory? Nelson. NIH Public Access, 6123(7), 323-338. http://doi.org/10.1016/S00796123(07)00020-9. What

3. Dunne, C., Henry Riche, N., Lee, B., Metoyer, R., \& Robertson, G. (2012). GraphTrail: Analyzing Large Multivariate, Heterogeneous Networks while Supporting Exploration History. Proceedings of the 2012 ACM Annual Conference on Human Factors in Computing Systems - CHI '12, 1663-1664. http://doi.org/10.1145/2207676.2208293

4. Feng, M., Deng, C., Peck, E. M., \& Harrison, L. (2017). HindSight: Encouraging Exploration through Direct Encoding of Personal Interaction History. IEEE Transactions on Visualization and Computer Graphics, 23(1), 351-360. http://doi.org/10.1109/TVCG.2016.2599058

5. Heer, J., Mackinlay, J. D., Stolte, C., \& Agrawala, M. (2008). Graphical Histories for Visualization : Supporting Analysis, Communication, and Evaluation, 14(6), 1189-1196.

6. Kadivar, N., Chen, V., Dunsmuir, D., Lee, E., Qian, C., Dill, J., ... Woodbury, R. (2009). CzSaw - Capturing and supporting the analysis process. VAST 09 - IEEE Symposium on Visual Analytics Science and Technology, Proceedings, 131-138. http://doi.org/10.1109/VAST.2009.5333020

7. Lamming, M. G. ., \& Newman, W. M. . (1992). Activity-based Information Retrieval: Technology in Support of Personal Memory. In Friedrich H. Vogt (Ed.), 12th World Computer Congress on Personal Computers and Intelligent Systems - Information Processing '92 (pp. 68-81). Amsterdam: North-Holland Publishing Co. Amsterdam, The Netherlands, The Netherlands (C1992.

8. Lenay, C., Thouvenin, I., Guénand, A., Gapenne, O., Stewart, J., \& Maillet, B. (2007). Designing the ground for pleasurable experience. Proceedings of the 2007 Conference on Designing Pleasurable Products and Interfaces - DPPI '07, (August), 35. http://doi.org/10.1145/1314161.1314165

9. Nguyen, P. H., Xu, K., Bardill, A., Salman, B., Herd, K., \& Wong, B. L. W. (2016). SenseMap: Supporting browser-based online sensemaking through analytic provenance. 2016 IEEE Conference on Visual Analytics Science and Technology, VAST 2016 Proceedings, (October), 91-100. http://doi.org/10.1109/VAST.2016.7883515

10. North, C., Chang, R., Endert, A., Dou, W., May, R., Pike, B., \& Fink, G. (2011). Analytic Provenance: Process+Interaction+Insight. CHI '11 Extended Abstracts on Human Factors in Computing Systems, 33-36. http://doi.org/10.1145/1979742.1979570

11. Pernice, K., \& Nielsen, J. (2009). How to Conduct Eyetracking Studies, (August), 159. Retrieved from http://media.nngroup.com/media/reports/free/How_to_Conduct_Eyetracking_Studies.pdf

12. Shneiderman, B. (1996). The eyes have it: a task by data type taxonomy for information visualizations. Proceedings 1996 IEEE Symposium on Visual Languages, 336-343. http://doi.org/10.1109/VL.1996.545307

13. Yang, L., Morizet-mahoudeaux, P., Guénand, A., \& Mouloudi, A. (2016). First steps towards the emergence of emotions in interaction design (p. 2016). 